gras qui n'est attaqué qu'en dernier. N'est-il pas admis, pour la Grenouille, que le développement des éléments sexuels et la résorption des appendices épiploïques sont en corrélation? La théorie de M. Pérez n'entraîne pas la conviction. Il sera toujours facile d'ergoter sur la signification, sur la valeur de tel ou tel trouble physiologique; mais dans un essai d'interprétation des métamorphoses, il nous semble impossible de ne pas tenir compte des troubles fonctionnels, en particulier des troubles circulatoires et respiratoires.

\title{
Nouveau Péripate des environs de Rio-de-Janeiro
}

Par E.-L. Bouvier.

Parmi les Onychophores qui font partie de la collection du Musée de Hambourg (1) se trouvent deux exemplaires d'un Peripatus que M. le $\mathrm{D}^{\mathrm{r}} \mathrm{Ohaus}$ a capturé tout récemment, à Petropolis, c'est-à-dire dans le voisinage immédiat de Rio-de-Janeiro. Ne fût-cequ'en raison du centre géographique qu'il habite, ce Péripate mériterait d'attirer l'attention; avec le Peripatoides chilensis $\mathrm{Blan} \mathrm{ch}$., il est le plus méridional de tous les Onychophores américains et se trouve séparé par environ 20 degrés de latitude des espèces les moins éloignées de l'Amérique du Sud orientale. Ces dernières ne paraissent pas, en effet, dépasser la région de l'Amazone : l'une est le Peripatus brasiliensis Bouv., de Santarem, l'autre le $P$. Simoni Bouv. qui a été trouvé aux environs de Caracas, et capturé depuis à l'embouchure de l'Amazone (coll. du British Museum).

Malgré ces différences de latitude, l'espèce du Musée de Hambourg appartient au même groupe de formes que les Péripates américains situés à l'est de la chaìne des Andes. Il se range, en d'autres termes, parmi les espèces que j'ai désignées sous le nom de Péripates caraïbes et il en présente tous les caractères $\left(^{2}\right)$. Il est du reste fort voisin des deux espèces de l'Amazone que j'ai citées plus haut; mais il se distingue du $P$. brasiliensis par les bifurcations segmentaires normales de ses plis dorsaux, de cette dernière espèce et du P. Simoni par l'absence à peu près complète de papilles accessoires. A ce point de vue, comme à tout autre égard, il ressemble surtout au P. Geayi Bouv., de la Guyane francaise, et se fait remarquer comme lui par la base

(1) Je profite de celte note pour présenter mes sincères remerciements à M. le professeur Kraepelin, qui a eu l'obligeance de me communiquer cette collection.

(2) E. - L. Bouvie r. Sur les variations et les groupements spécifiques des Péripates américains. Compt. rend. Acad. Sc., t. 128, p. 1344, 1899. 
quadrangulaire de ses papilles principales ainsi que par son corps grêle et allongé. Il a du reste des caractères propres qui permettent de ne pas le confondre avec cette dernière espèce : papilles principales primaires subcylindriques et d'ailleurs médiocrement élevées, papilles principales secondaires un peu moins nombreuses que les précédentes et s'intercalant isolément entre elles, sillons de séparation des papilles bien moins distincts que dans l'espèce de Geay. Les papilles secondaires ont des dimensions variables, mais sont toujours assez grandes et parfois même ne se distinguent pas des papilles principales. Le plus souvent, elles sont beaucoup plus longues que larges et se présentent alors sous la forme d'une saillie conique dont la base s'allonge en rectangle; sur cette base s'élèvent, dans quelques cas fort rares, une ou deux papilles accessoires, de sorte que le rectangle intercalaire rappelle un peu les groupements rectangulaires de papilles qu'on observe dans le $\boldsymbol{P}$. imthurmi Scl. Le nombre des pattes est aussi réduit que dans les exemplaires de cette dernière espèce les moins bien doués sous ce rapport : il est de 27 paires dans l'un des exemplaires femelles qui m'ont été soumis et de 28 dans l'autre ( $\left.{ }^{1}\right)$. Ce nombre peut s'élever à 31 paires dans le $\boldsymbol{P}$. imthurmi; il est de 31 paires dans le $\boldsymbol{P}$. brasiliensis, de 32 paires dans le $\boldsymbol{P}$. Geayi et de 31 ou 32 paires dans le $\boldsymbol{P}$. Simoni.

Je désignerai cette espèce sous le nomde $\mathbf{P}$. Ohausi, n. sp., en l'honneur de notre excellent confrère, $\mathrm{M}$. le $\mathrm{D}^{\mathrm{r}} \mathrm{Ohaus}$, qui l'a capturée.

La découverte du $P$. Ohausi nous permet de constater combien est homogène le groupe des Péripates caraïbes et combien sont étroites les affinités des espèces, déjà nombreuses, qui le représentent. Ces affinités subissent, du reste, des modifications progressives, qui paraissent être, pour la plupart, sous la dépendance étroite de l'aire géographique; c'est ainsi que le $P$. Ohausi se rapproche surtoat des espèces américaines les plus voisines. Le groupe des Péripates andicoles n'est pas moins homogène; pourtant il s'est modifié plus vite quand changeaient les latitudes et, dans les régions septentrionales du Chili, se trouve déjà représenté, d'après M. Silvestri (Zool. Anz., 1899), par des Peripatoides, c'est-à-dire par des formes que l'on avait crues jusqu'ici localisées en Nouvelle-Zélande. Pour trouver des modifications analogues dans le groupe des Péripates caraibes, il faut quitter l'Amérique et atteindre l'Afrique occidentale. Au Congo, en effet, le genre

(1) Ces deux exemplaires ont sensiblement 43 mill. de longueur, 4 de largeur et 3 d'épaisseur; leurs utérus paraissent vides, mais leurs réceptacles séminaux sont blancs et probablement remplis de spermatozoïdes. 
Peripatus est représenté par une curieuse espèce, le $\boldsymbol{P}$. Tholloni Bouv., qui se rapproche des Péripates caraïbes par la grande majorité de ses caractères, mais qui n'est pas sans présenter aussi quelques affinités avec les Peripatopsis de l'Afrique australe.

Pour terminer cette rapide revue du groupe, j'ajouterai que ces derniers, au moins par leur mode de développement, se rapprochent des Paraperipatus, de la Nouvelle-Bretagne. Si la plupart d'entre eux ( $\boldsymbol{P}$. capensis Grube, $\boldsymbol{P}$. Balfouri Sedgw.) se font remarquer par l'uniformité du développement de leurs embryons et par la nutrition purement ectodermique de ces derniers, j'ai pu me convaincre que les femelles de plusieurs Peripatopsis renferment dans leur intérieur des embryons diversement développés et que, dans le $P$. Sedgwicki Purc., ces embryons, à un certain stade, présentent dans la partie antérieure de leur région dorsale une vésicule embryonnaire qui ressemble, abstraction faite des dimensions, à l'énorme vésicule trophique signalée par M. Willey dans le Paraperipatus Novae-Britanniae Willey. J'ajouterai que M. Purcell a signalé des embryons à divers stades dans l'Opisthopatus cinctipes Purc., du Natal, et que j'ai pu vérifier cette observation sur des femelles appartenant à une variété de cette dernière espèce.

\section{Bulletin bibliographique.}

Académie des Sciences (C. R. hebdom. des Séances), 1900, I, 4-6. - $\odot$ Annales des Sciences naturelles, IV, 1820\%. - L. Dufour : Recherches anatomiques sur les Carabiques et sur plusieurs autres Coléoptères (suite). - Recherches anatomiques sur l'Hippobosque des Chevaux. - J. Hayenbach : Description du Mormolyce, nouveau genre d'Insecte dans l'ordre des Coléoptères.

Annals and Magazine of Natural History (The), février 1900. - Miss G. Ricardo : Notes on the Pangoninae of the Family Tabanidae in the British Museum Collection. - A. Goldsborough Mayer: On the Mating Instinct in Moths. - C. Norman : British Amphipoda : Fam. Lysianassidae (pl.). - G. LewIs : On new Species of Histeridae and Notices of others. - E. Bordage : On the Absence of Regeneration in the posterior Limbs of the Orthoptera Saltatoria and ist probable Causes. - Regeneration of the Tarsus and of the two anterior Pairs of Limbs in the Orthoptera Saltatoria. 


\section{$2 \mathrm{BHL}$ Biodiversity Heritage Library}

Bouvier, E.-L. 1900. "Nouveau Péripate des environs de Rio-de-Janeiro." Bulletin de la Société entomologique de France 1900, 66-68.

View This Item Online: https://www.biodiversitylibrary.org/item/36399

Permalink: https://www.biodiversitylibrary.org/partpdf/37066

\section{Holding Institution}

Smithsonian Libraries

\section{Sponsored by}

Smithsonian

\section{Copyright \& Reuse}

Copyright Status: NOT_IN_COPYRIGHT

This document was created from content at the Biodiversity Heritage Library, the world's largest open access digital library for biodiversity literature and archives. Visit BHL at https://www.biodiversitylibrary.org. 